\title{
Critical Kerr nonlinear optical cavity in the presence of internal loss and driving noise
}

\author{
André Thüring* and Roman Schnabel \\ Max-Planck-Institut für Gravitationsphysik (Albert-Einstein-Institut) and Institut für Gravitationsphysik, Leibniz Universität Hannover, \\ Callinstrasse 38, D-30167 Hannover, Germany \\ (Received 21 December 2010; published 20 September 2011)
}

\begin{abstract}
We theoretically analyze the noise transformation of a high-power continuous-wave light field that is reflected off a critical Kerr nonlinear cavity (KNLC). Our investigations are based on a rigorous treatment in the time domain. Thereby, realistic conditions of a specific experimental environment including optical intracavity loss and strong classical driving noise can be modeled for any KNLC. We show that, even in the presence of optical loss and driving noise, considerable squeezing levels can be achieved. We find that the achievable squeezing levels are not limited by the driving noise but solely by the amount of optical loss. Amplitude-quadrature squeezing of the reflected mean field is obtained if the KNLC's operating point is chosen properly. Consistently, a KNLC can provide a passive purely optical reduction of laser-power noise as experimentally demonstrated in Khalaidovski et al. [Phys. Rev. A 80, 053801 (2009)]. We apply our model to this experiment and find good agreement with measured noise spectra.
\end{abstract}

DOI: 10.1103/PhysRevA.84.033839

PACS number(s): 42.65.Hw, 42.50.Lc, 42.79.Gn

\section{INTRODUCTION}

Due to the effect of self-squeezing, the (quantum-) noise distribution of a light field can be manipulated when passed through a Kerr medium [1] or when coupled to and reflected off a Kerr nonlinear cavity (KNLC) (Fig. 1) [2-5]. In this way, the optical Kerr effect can be used for the generation of squeezed light, which can be exploited to enhance stateof-the-art metrology to quantum metrology. For a review, we refer to Ref. [6]. In contrast to methods based on secondorder nonlinear processes-such as the optical-parametric oscillation-no second-harmonic pump field is required for operating the KNLC. Thus, a technical realization of squeezed light generation seems to be less involved. It was shown by Collet and Walls [3] that a lossless KNLC, operated at its so-called critical point (refer to the centered curves in Fig. 2), provides perfect squeezing in the amplitude quadrature of the reflected field at zero Fourier frequency. This specific characteristic enables a further technical application in the classical noise regime: In contrast to conventional techniques [7,8], a KNLC can provide a light-power stabilization within its bandwidth and in a purely optical passive way, as observed in Ref. [9]. Such a passive classical noise-reduction scheme might find an application in gravitational-wave astronomy. Here, ultrastable high-power laser radiation is needed [10] to achieve a sufficiently high interferometric sensitivity, e.g., as envisioned for Advanced LIGO [11] and the Einstein Telescope [12].

Reynaud et al. [13] have analyzed the squeezing of quantum noise achievable in the reflection of a multistable KNLC. They showed that a KNLC, operated close to the turning points of the corresponding resonance curve, also yields perfect squeezing at zero Fourier frequency, although not in the amplitude quadrature of the reflected field. In fact, they revealed that the amplitude quadrature at zero frequency is not squeezed if a lossless multistable KNLC is considered.

\footnotetext{
*Andre.Thuering@aei.mpg.de
}

Consistently, the multistable state is not favorable if a passive purely optical reduction of laser-power noise is targeted at low frequencies.

In the pulsed-laser regime, the squeezing of quantum noise by means of the optical Kerr effect was demonstrated in several experiments $[14,15]$. In these experiments, no optical cavities were involved. The reduced quantum noise was observed in certain field quadratures that depart from the amplitude quadrature of the mean field. Also, in the continuous-wave laser regime, one experiment was conducted to demonstrate squeezing of quantum noise [16]. Here, a small reduction of about $1.5 \mathrm{~dB}$ below the shot-noise limit could be demonstrated by means of a KNLC driven by a 1.2-mW laser beam. White et al. [17] have used a KNLC pumped by a 30-mW laser beam and achieved a reduction by $1.5 \mathrm{~dB}$ of classical noise. Recently, at an intermediate-power 0.75 -W laser, a classical power-noise reduction by a surprisingly great factor of $32 \mathrm{~dB}$ by means of a KNLC was demonstrated [9]. This strong reduction was observed at the driving laser's relaxation oscillation frequency where its noise in the amplitude quadrature is much higher than in the phase quadrature.

In this paper, we report on our numerical investigations of the noise transformation of a high-power light field that is coupled to and reflected off a critical KNLC. We used the approach of a rigorous treatment in the time domain, which easily includes the full nonlinear formalism and the full range of different experimental parameters, such as low- and high-cavity finesse values. Thus, it is also possible to calculate the cavity dynamics on time scales of the cavity storage time revealing cavity ringing or the hysteresis effect as well as the nonlinear response to large (classical) signals, refer, e.g., to Figs. 6.15 and 6.17 in Ref. [18], respectively. Here, we use our model to describe the squeezing of quantum noise and weak classical driving noise with Gaussian statistics under consideration of the KNLC's detuning and loss. Model input parameters are the cavity length, the cavity mirror reflectivities, the intracavity optical loss, the Kerr nonlinearity, and the optical pump power. As all of these parameters can be determined or, at least, can be estimated in experimental environments, our 


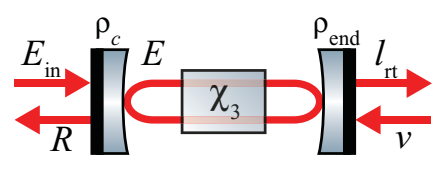

FIG. 1. (Color online) Schematic of a KNLC. $E_{\text {in }}$ is the driving field coupled to the cavity, $E$ is the intracavity field, $l_{\mathrm{rt}}$ is the fraction of $E$ that is lost per round-trip at the imperfect end mirror, and $v$ is the vacuum field that couples in at the end mirror. The reflected field is denoted as $R$. We investigated the regime where $\rho_{\mathrm{c}}<\rho_{\text {end }} \leqslant 1$.

numerical calculations have a great potential to easily model and to understand experimental results, e.g., those reported in Ref. [9].

In the following, the noise distribution in phase space is analyzed for a quantum-noise-limited driving field as well as for a field that shows (unbalanced and possibly correlated) classical noise in its amplitude and phase quadratures. In both cases, several values for the internal cavity loss are considered, and for certain operating points, the noise transformation is illustrated by the corresponding Wigner functions [19]. We focus on the noise reduction in the amplitude quadrature of the mean field that is reflected off the KNLC. This is essential in view of a potential passive purely optical reduction of laser-power noise. We show that the presence of intracavity loss strongly influences the phase-space rotation and, thus, the quadrature yielding the optimal noise reduction. Additionally, we show that, even for a driving field with an unbalanced (classical) noise distribution in its amplitude and phase quadratures, strong noise reductions, even below quantum noise, can be achieved. As already shown by Collet and Walls [3], for a quantum-noise-limited driving field, the lossless KNLC needs to be operated at its critical point in order to obtain optimal squeezing (noise reduction) in the amplitude quadrature of the reflected mean field. We show that this condition still holds for a driving field that exhibits classical noise in both amplitude and phase quadratures. In the presence of intracavity loss, however, amplitude-quadrature noise reduction can be obtained with a critical KNLC only if operated with a detuning aside from the critical point.

\section{CALCULATION OF THE LIGHT FIELDS}

The nonlinear nature of a KNLC becomes evident by looking at the analytic expression for the monochromatic steady-state intracavity field (for zero loss) as given by

$$
E=\frac{i \tau_{\mathrm{c}}}{1-\rho_{\mathrm{c}} \rho_{\mathrm{end}} \exp \left[2 i\left(\Phi+\theta|E|^{2}\right)\right]} E_{\mathrm{in}} .
$$

Here, $\rho_{\mathrm{c}}\left(\tau_{\mathrm{c}}\right)$ and $\rho_{\text {end }}\left(\tau_{\text {end }}\right)$ are the amplitude reflectance (transmittance) factors of the coupling and end mirror, respectively. $\Phi$ denotes the geometric detuning with respect to the carrier light frequency $\omega_{0}$. Furthermore, $\theta|E|^{2}$ is the intensity-dependent phase shift induced by the optical Kerr effect. In the variable $\theta$, the nonlinear refraction index $n_{2}$, the length of the Kerr medium $L_{\mathrm{KM}}$, the cross-sectional area $\mathcal{A}$ of the light field, the speed of light $c$, and the carrier frequency $\omega_{0}$ are included according to [5]

$$
\theta=\frac{n_{2} \omega_{0} L_{\mathrm{KM}}}{2 \mathcal{A} c}
$$
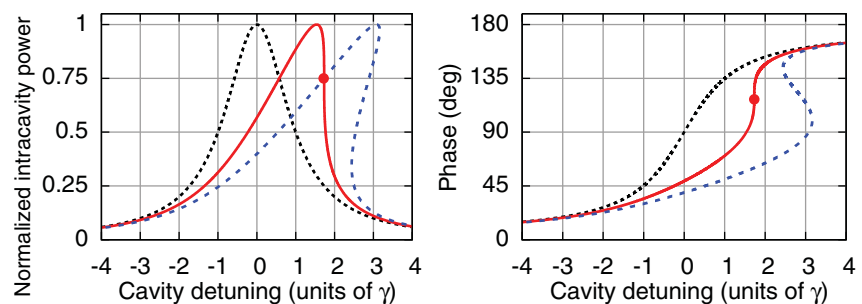

FIG. 2. (Color online) Normalized intracavity power (left) and phase (right) without Kerr effect (black dotted curves), the so-called critical state (red solid curves) with its critical point of infinite slope (highlighted by the red circles), and a multistable state (blue dashed curves) of the KNLC with half-bandwidth $\gamma$.

Figure 2 shows the intracavity power $P=|E|^{2}$ depending on the (static) cavity detuning $\Phi$ for three cases: the linear case with $\theta=0$ (black dotted curve), the so-called critical state with $\theta=\theta_{\text {crit }}$ (red solid curve), and a multistable case with $\theta=2 \theta_{\text {crit }}$ (blue dashed curve). Because Eq. (1) is a transcendental (implicit) equation, it has been solved numerically. Figure 2 reveals that the critical state and the multistable state exhibit particular operating points at which the resonance curve shows an infinite slope. In the critical state, the infinite slope occurs at the critical operating point (OP) $\mathrm{OP}_{\text {crit }}$ (highlighted by the red circles) and in the multistable state at the two turning points of the resonance curve. As shown in Refs. [3] and [13], these operating points are favorable in view of achieving high squeezing-factors. However, since in the multistable case, perfect squeezing is not obtained in the amplitude quadrature of the reflected field, we restrict our investigations to the critical state.

In order to model noisy optical fields reflected off the KNLC in the time domain, it is necessary to evaluate the light fields (and their interference) after each round-trip. Because we are interested in the noise transfer of a KNLC set to a certain operating point, we assume a static detuning of the cavity, i.e., $\Phi(t)=\Phi=$ constant. Following this line, the internal field reads

$$
E\left(n t_{\mathrm{rt}}\right)=i \tau_{\mathrm{c}} E_{\mathrm{in}}\left(n t_{\mathrm{rt}}\right)+E_{\mathrm{rt}}\left[(n-1) t_{\mathrm{rt}}\right]
$$

Here,

$$
\begin{aligned}
E_{\mathrm{rt}}\left[(n-1) t_{\mathrm{rt}}\right]= & \rho_{\mathrm{end}} \rho_{\mathrm{c}} \exp \left\{2 i \Phi+2 i \theta\left|E\left[(n-1) t_{\mathrm{rt}}\right]\right|^{2}\right\} \\
& \times E\left[(n-1) t_{\mathrm{rt}}\right]
\end{aligned}
$$

describes the field after one round-trip. The nonlinearity of our approach at this stage is evident due to the exponential function in Eq. (4) depending on the field intensity $|E|^{2}$. The round-trip time in a cavity of length $L$ is

$$
t_{\mathrm{rt}}=\frac{2 L}{c} .
$$

The reflected field is then given by

$$
R\left(n t_{\mathrm{rt}}\right)=\rho_{\mathrm{c}} E_{\mathrm{in}}\left(n t_{\mathrm{rt}}\right)+i \tau_{\mathrm{c}} E\left(n t_{\mathrm{rt}}\right) .
$$

The time dependence of the input field can be described by

$$
E_{\text {in }}(t)=\overline{E_{\text {in }}}+\delta E(t)_{\text {in }}, \quad \text { with } \overline{\delta E_{\text {in }}(t)}=0 .
$$


If the mean field $\overline{E_{\text {in }}}$ is set to be real, Eq. (7) can be written as

$$
E_{\mathrm{in}}(t)=E_{0}+\delta X_{1}(t)+i \delta X_{2}(t),
$$

giving a real-valued phase-space description of the input laser field with $\delta X_{1}(t)$ and $\delta X_{2}(t)$ being the fluctuations in its amplitude $\left(X_{1}\right)$ and phase quadratures $\left(X_{2}\right)$, respectively. Then, the same description does apply to the reflected field after $n$ round-trips according to Eq. (6) and its converged value for $n \Rightarrow \infty$.

Eventually, the cavity-reflected fluctuating fields of our converged time-domain simulations need to be expressed as normalized noise spectral densities in order to allow for a comparison with experimental data. For this reason, we apply a fast Fourier transformation (FFT) to the reflected field and normalize the result such that, for a zero Kerr effect, the vacuum noise of a coherent input field transforms into a noise spectral density of magnitude unity $(0 \mathrm{~dB})$ with a white spectrum.

In the following, we describe our time-domain simulation in more detail. We also describe the transfer functions we used to calculate the noise spectral densities for other than vacuumnoise input, i.e., for classical noise inputs with unbalanced, possibly correlated, fluctuations in the amplitude and phase quadratures. With respect to the transfer functions, we restrict ourselves to the regime where the noise amplitude is much smaller than the driving field amplitude, i.e., to the regime where a linearized approximation is valid. For all results presented here, a fluctuation at a certain Fourier frequency is always transformed into output noise at the same single frequency, since the Fourier transformation does not show any additional frequency components. Note that we indeed have observed additional frequency components for parameters outside the regime presented in this paper.

We consider an amplitude-modulated input field by setting Eq. (8) to

$$
E_{\text {in }}=E_{\mathrm{am}}(t)=E_{0}+x_{1} \cos (\Omega t),
$$

and accordingly to

$$
E_{\mathrm{in}}=E_{\mathrm{pm}}(t)=E_{0}+i x_{2} \cos (\Omega t)
$$

for a phase-modulated field. Here, $\Omega$ is the angular Fourier frequency and $x_{1}$ and $x_{2}$ are real-valued scaling factors with $\left|x_{1}\right|,\left|x_{2}\right| \ll\left|E_{0}\right|$. The FFT (we have used FFTW3 [20]) of the reflected field $R\left(n t_{\mathrm{rt}}\right)$ provides the relative amplitudes of upper and lower sidebands at positive and negative frequencies $R( \pm \Omega)$, respectively. The phases of upper and lower sidebands determine whether an input amplitude modulation is transferred to an output amplitude or a phase modulation. The full coupling can be described by four coefficients $T_{i j}$,

$$
\begin{aligned}
& T_{11}=\left[R_{\mathrm{am}}^{*}(-\Omega)+R_{\mathrm{am}}(\Omega)\right] /\left(2 x_{1}\right), \\
& T_{12}=\left[R_{\mathrm{pm}}^{*}(-\Omega)+R_{\mathrm{pm}}(\Omega)\right] /\left(2 x_{2}\right), \\
& T_{21}=\left\{i\left[R_{\mathrm{am}}^{*}(-\Omega)-R_{\mathrm{am}}(\Omega)\right]\right\} /\left(2 x_{1}\right), \\
& T_{22}=\left\{i\left[R_{\mathrm{pm}}^{*}(-\Omega)-R_{\mathrm{pm}}(\Omega)\right]\right\} /\left(2 x_{2}\right) .
\end{aligned}
$$

These coefficients can be written as a $2 \times 2$ matrix $\mathbf{T}(\Omega)$, which is commonly referred to as the (linearized) input-output transfer function. The spectral density matrix (covariance matrix) reads

$$
\mathbf{S}(\mathbf{T})=\frac{1}{2}\left(\mathbf{T} \cdot \mathbf{T}^{\dagger}+\mathbf{T}^{*} \cdot \mathbf{T}^{\mathrm{T}}\right) .
$$

The diagonal components of the $2 \times 2$ matrix $\mathbf{S}$ correspond to the normalized power spectral densities of the field's amplitude $\left(X_{1}\right)$ and phase $\left(X_{2}\right)$ quadrature amplitudes. Offdiagonal components are due to correlations between the two quadratures. From this matrix, the spectral density of any measured linear combination $X_{\zeta}=\cos (\zeta) X_{1}+\sin (\zeta) X_{2}$ can be evaluated as

$$
S_{\zeta}=\left(\begin{array}{ll}
\cos \zeta & \sin \zeta
\end{array}\right) \cdot \mathbf{S}(\mathbf{T}) \cdot\left(\begin{array}{c}
\cos \zeta \\
\sin \zeta
\end{array}\right),
$$

where $\zeta$ denotes the homodyning angle. If the noise transformation is considered for other than the reference vacuum input, the matrix $\mathbf{T}$ needs to be replaced by

$$
\mathbf{T}^{\prime}=\mathbf{T} \cdot\left(\begin{array}{cc}
\cos \vartheta & -\sin \vartheta \\
\sin \vartheta & \cos \vartheta
\end{array}\right) \cdot\left(\begin{array}{cc}
\exp \left(2 r_{1}\right) & 0 \\
0 & \exp \left(2 r_{2}\right)
\end{array}\right) .
$$

The matrix on the right is the general squeezing matrix with $\left(r_{1}+r_{2}\right) \geqslant 0$ due to Heisenberg's uncertainty relation. Values $r_{1,2}<0$ correspond to a noise level below vacuum noise (squeezed), and $r_{1,2}>0$ correspond to a noise level above vacuum noise (antisqueezed), respectively. The matrix on the left describes a rotation of the squeezing (noise) ellipse in phase space, i.e., the squeezed quadrature is determined by the squeezing angle $\vartheta$. In order to constitute a driving field that exhibits stationary classical noise (such as thermal noise) in its amplitude and phase quadratures, both $r_{1}$ and $r_{2}$ need to be greater than zero. For an extensive overview of this linear transfer function formalism, we refer to Ref. [21].

In addition to quantum noise and classical driving noise that enters the KNLC through the coupling mirror, vacuum-noise contributions due to intracavity loss also need to be considered, and the corresponding input-output relation (denoted as $\mathbf{L}$ in the following) needs to be derived. For simplicity, we map the cavity round-trip loss $l_{\mathrm{rt}}$ onto the end-mirror amplitude transmissivity $\tau_{\mathrm{end}}=l_{\mathrm{rt}}$. Hence, similar to the derivation of the input-output relation $\mathbf{T}$, we consider the transfer function for the following light fields:

$$
\begin{gathered}
v_{\mathrm{am}}(t)=x_{1} \cos (\Omega t), \\
v_{\mathrm{pm}}(t)=i x_{2} \cos (\Omega t),
\end{gathered}
$$

that couple in at the end mirror. The beat with the driving field is described by setting $E_{\text {in }}(t)$ to $E_{0}$, and the time-dependent loss-driven amplitude modulation inside the cavity then reads

$$
\begin{aligned}
E_{\mathrm{am}}\left(n t_{\mathrm{rt}}\right)= & i \tau_{\mathrm{end}} v_{\mathrm{am}}(t)+i \tau_{\mathrm{c}} E_{0} \exp \left\{i \Phi_{\mathrm{opt}}\left[(n-1) t_{\mathrm{rt}}\right]\right\} \\
& +E_{\mathrm{rt}, \mathrm{am}}\left[(n-1) t_{\mathrm{rt}}\right] .
\end{aligned}
$$

The contribution to the overall noise is given by the KNLC transmitted part, which reads

$$
T\left(n t_{\mathrm{rt}}\right)=i \tau_{\mathrm{c}} E_{\mathrm{am}}\left(n t_{\mathrm{rt}}\right) \exp \left\{\Phi_{\mathrm{opt}}\left[(n-1) t_{\mathrm{rt}}\right]\right\} .
$$

In analogy to Eqs. (11)-(14), the coefficients of the inputoutput transfer function $\mathbf{L}(\Omega)$ are determined from the FFT 
of $T\left(n t_{\mathrm{rt}}\right)$. Finally, the total-power spectral density matrix is given by

$$
\mathbf{S}_{\mathrm{tot}}(\Omega)=\mathbf{S}(\mathbf{T}(\Omega))+\mathbf{S}(\mathbf{L}(\Omega))
$$

\section{AMPLITUDE-QUADRATURE SQUEEZING}

First, we analyze a shot-noise-limited (vacuum-noiselimited $r_{1}=r_{2}=0$ ) input field and calculate the power-noise spectra for several values of the KNLC round-trip loss $l_{\mathrm{rt}}^{2}$. It is convenient to quantify the loss in terms of the so-called escape efficiency,

$$
\eta_{\mathrm{esc}}=\frac{\tau_{\mathrm{c}}^{2}}{l_{\mathrm{rt}}^{2}+\tau_{\mathrm{c}}^{2}} \quad \Leftrightarrow \quad l_{\mathrm{rt}}^{2}=\frac{\tau_{\mathrm{c}}^{2}\left(1-\eta_{\mathrm{esc}}\right)}{\eta_{\mathrm{esc}}} .
$$

We consider escape efficiencies of 1 (lossless), 0.999, 0.99, 0.9 , and 0.75 , respectively. In all cases, the KNLC is set to its critical point, and the power-noise spectrum is calculated for the $X_{1}$ quadrature of the reflected mean field $\left(S_{11, \text { tot }}\right)$. The results are shown in Fig. 3. The spectrum obtained for the lossless case agrees with the results of Collet and Walls [3] and Rehbein et al. [5] and yields the predicted squeezing in the amplitude quadrature. If a KNLC with internal optical loss $\left(\eta_{\mathrm{esc}}<1\right)$ is considered, the spectra show a qualitatively different behavior. The intracavity loss affects the phase-space rotation of the reflected light field such that the squeezed quadrature deviates from the amplitude quadrature. This deviation increases with increasing loss as can be seen in the lower graph of Fig. 3. Please, also refer to the Wigner functions obtained for the critical point $\left(\mathrm{OP}_{6, \text { crit }}\right)$ shown in Figs. 6 and 7. Correspondingly, for comparatively low-escape efficiencies (cyan long dashed-dotted and yellow dashed-dotted curves in Fig. 3), the beam reflected off the KNLC shows a considerably enhanced amplitude-quadrature noise at low frequencies. Around the pole frequency of the KNLC, the cavity dispersion turns the noise ellipse and, thus, the squeezed quadrature toward the amplitude quadrature of the reflected light field. As the (anti)squeezing levels degrade at frequencies above the cavity's bandwidth, only moderate squeezing levels can be achieved around $\Omega=\gamma$. In the case of high-escape efficiencies ( $\eta_{\text {esc }}=0.999$ and 0.99 ), considerable amplitude-quadrature squeezing can still be achieved in the midfrequency range. On the contrary, at low frequencies where the (anti)squeezing factors are comparatively high, a small deviation of the squeezed quadrature from the amplitude quadrature already leads to a degraded squeezing level or even a noise enhancement, respectively.

Likewise, we analyze the noise transformation for an input field that still has Gaussian statistics but exhibits an unbalanced noise distribution in phase space. To constitute a realistic situation of an experiment, we choose the noise of the driving field in its $X_{\vartheta}$ quadrature with $20 \mathrm{~dB}$ and in its $X_{\vartheta+90^{\circ}}$ quadrature with $10 \mathrm{~dB}$ above the reference value. The quadrature angle $\vartheta$ is set to $40^{\circ}$ with respect to the $X_{1}$ quadrature of the driving mean field. The results obtained are shown in Fig. 4. In the lossless case $\left(\eta_{\mathrm{esc}}=1\right)$, the relative spectral noise reduction is essentially the same as in Fig. 3. At high frequencies, a slightly enhanced noise level appears. Here, the (anti)squeezing levels are almost zero, but the cavity dispersion causes a rotation of the initial noise ellipse (refer

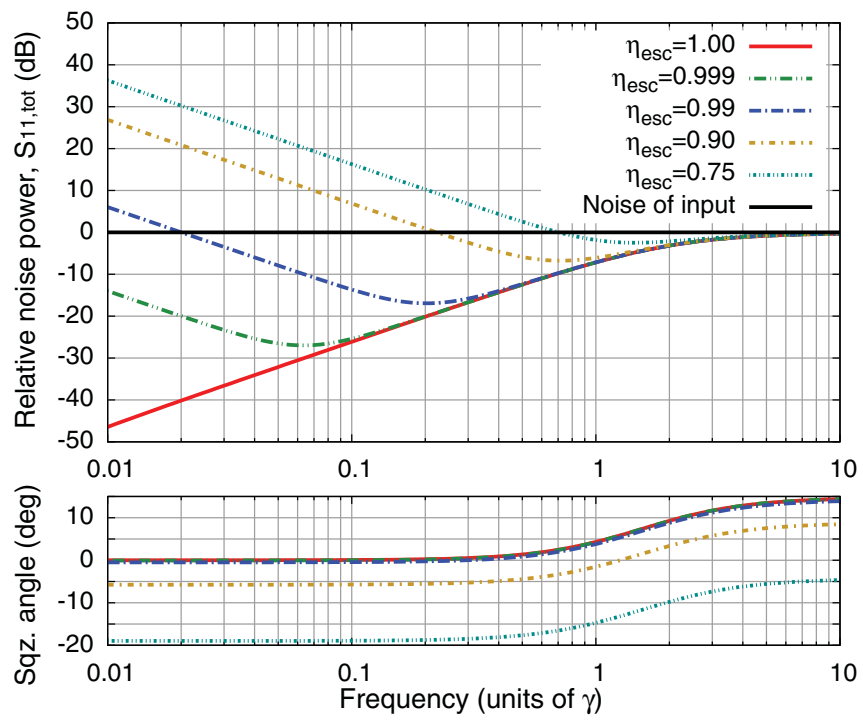

FIG. 3. (Color online) (Top) Amplitude-quadrature noise spectra of the light field reflected off the critical KNLC for a shot-noiselimited input field $(0-\mathrm{dB}$ reference). The frequency is normalized to the respective cavity half-bandwidth $\gamma$. Five values for the escape efficiency $\eta_{\text {esc }}$ are considered. In all cases, squeezing of quantum noise can be observed. (Bottom) The graph shows the frequency-dependent squeezing angle. Only for the lossless case does the squeezed quadrature match the $X_{1}$ quadrature $\left(0^{\circ}\right)$ at low frequencies. In the presence of intracavity loss, the squeezed quadrature deviates from the $X_{1}$ quadrature. This deviation increases with increasing loss.

to the lower graph) such that the $X_{\vartheta}$ quadrature approximates the $X_{1}$ quadrature of the reflected mean field. Please note that, at the lower bound of the considered spectrum, the noise reduction is about $45 \mathrm{~dB}$ just as in the case of a shot-noise-limited driving field (refer to Fig. 3). The squeezing is still about $30 \mathrm{~dB}$, although the driving field exhibits significant classical noise in both of its quadratures. In contrast to Fig. 3 , the spectra obtained for a KNLC with $\eta_{\text {esc }} \neq 1$ show narrow dips that fall below the noise level achieved in the lossless case. This feature becomes evident by looking at the middle graph of Fig. 4. It shows the frequency dependence of the quadrature that yields the lowest noise level. The dips in the spectra occur at that frequency where the squeezed quadrature coincides with the $X_{1}$ quadrature (i.e., with $0^{\circ}$ ) of the reflected mean field. This fact is highlighted by the arrows pointing from the middle toward the top graph.

\section{INFLUENCE OF THE CAVITY OP}

The foregone investigations show that, for intracavity loss $\left(\eta_{\text {esc }}<1\right)$, the squeezed quadrature does not coincide with the $X_{1}$ quadrature of the reflected mean field at low frequencies. It is obvious that this quadrature angle must have a connection with the chosen OP of the KNLC. Since we want to prove the KNLC's capability for a passive purely optical reduction laser-power noise under the consideration of a realistic experimental situation, we investigate the noise transformation in the dependence of the OP. In view of a possible application in a high-power-laser stabilization, e.g., for advanced gravitational-wave detectors [11,12], a noise reduction at frequencies down to $1 \mathrm{~Hz}$ are required. 


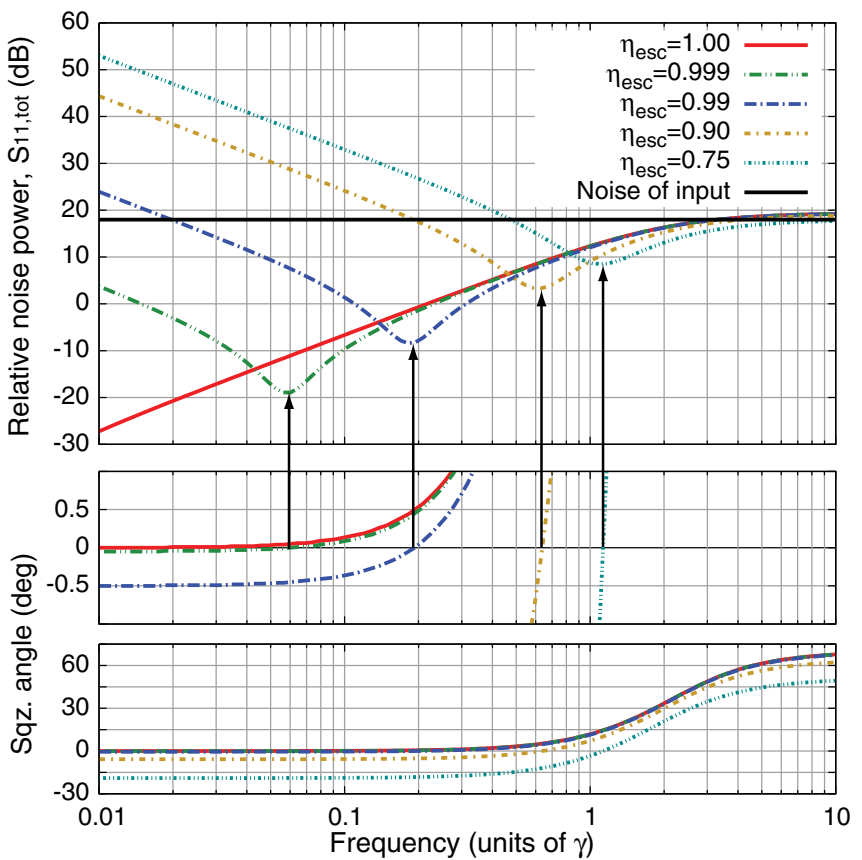

FIG. 4. (Color online) (Top) Amplitude-quadrature noise spectra of the KNLC as shown in Fig. 3 but for an input field with a classical and unbalanced noise distribution in quadrature phase space. The noise power in the $X_{\vartheta}$ quadrature and in the $X_{\vartheta+90^{\circ}}$ quadrature is set to 20 and $10 \mathrm{~dB}$, respectively, above shot noise. The quadrature angle $\vartheta$ is set to $40^{\circ}$. (Middle, bottom) The frequency-dependent quadrature angle yielding the lowest noise level. The middle graph shows a zoom of the bottom graph to make the origin of the dips in the spectra obtained for $\eta_{\text {esc }} \neq 1$ more obvious (see text). At low frequencies, this quadrature angle is almost the same as in Fig. 3. At frequencies far above the half-bandwidth $\gamma$, the squeezing factors are comparatively small, but the initial noise distribution is still rotated in phase space due to the detuned KNLC.

Thus, an optimization with regard to the noise reduction at low frequencies is favorable. We investigate the noise transformation for eight OPs at certain frequencies $(\Omega=0.1 \gamma$ and $\Omega=\gamma$, where $\gamma$ is the KNLC's half-bandwidth). The considered OPs lie on the steep resonance slope of the KNLC as illustrated in the left graph of Fig. 5. They correspond to $0.25-0.95$ of the intracavity power $P_{\text {res }}$ that is achieved on resonance. In all cases, the critical point relates to $\mathrm{OP}_{6 \text {, crit }}$ yielding an intracavity power of $0.75 P_{\text {res }}$ [22].

Generally, the modulation state of a light field at Fourier frequency $\Omega$ is fully characterized by its quasiprobability distribution in the quadrature-amplitude phase space-the so-called Wigner function. Plotted for several OPs, the Wigner function nicely illustrates the different noise transformations possible for a KNLC. Within the linearized approximation, Gaussian input noise is generally transformed into noise, again, with Gaussian statistics. For such states, the corresponding Wigner function is determined by the maximal and minimal noise levels (in the two orthogonal quadratures) and the orientation of the noise ellipse in phase space. One obtains

$$
W=\frac{1}{\pi} \exp \left[-x_{1, \vartheta}^{2} \exp \left(2 r_{1}\right)-x_{2, \vartheta}^{2} \exp \left(2 r_{2}\right)\right]
$$
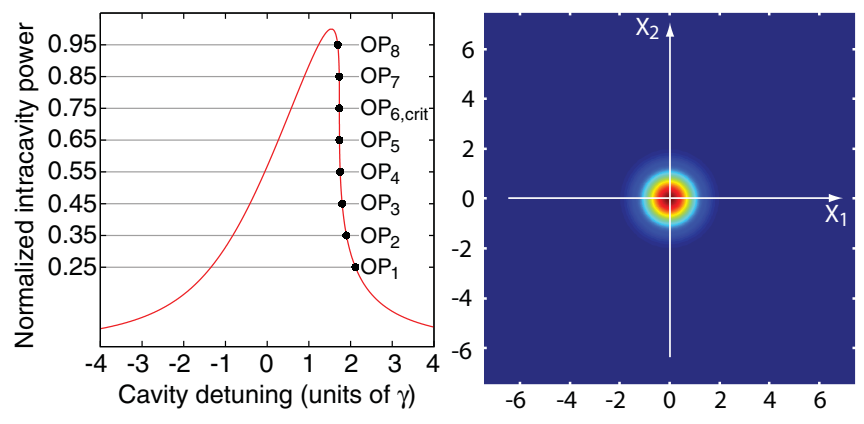

FIG. 5. (Color online) (Left) The graph shows eight OPs on the steep resonance slope of a critical KNLC. The operating point $\mathrm{OP}_{6 \text {,crit }}$ is the critical point. For all OPs, the noise transformations are investigated in phase space at sideband frequencies corresponding to $0.1 \gamma$ and $\gamma$, respectively. (Right) The graph shows the Wigner function of a vacuum state providing a reference for our analysis. Note that all modulation fields at sideband frequencies of a monochromatic coherent-state carrier field are, per definition, in a vacuum state. The $X_{1}$ axis corresponds to the amplitude quadrature of the carrier field, and the $X_{2}$ axis corresponds to its phase quadrature, respectively.

with

$$
\begin{aligned}
& x_{1, \vartheta}=x_{1} \cos \vartheta-x_{2} \sin \vartheta, \\
& x_{2, \vartheta}=x_{1} \sin \vartheta+x_{2} \cos \vartheta .
\end{aligned}
$$

The factors $\exp \left(2 r_{1,2}\right)$ account for a squeezed $\left(r_{1,2}<0\right)$ or an antisqueezed noise $\left(r_{1,2}>0\right)$. For $r_{1,2}=0$, one obtains the Wigner function of a vacuum state as shown in the right graph of Fig. 5. The Wigner function of a pure $10-\mathrm{dB}$ amplitudesqueezed state is determined by $r_{1}=\ln (0.1) / 2, r_{2}=\ln (10) / 2$, and $\vartheta=0$.

We note that Kitagawa and Yamamoto [1] and Reynaud et al. [13] showed that non-Gaussian Wigner functions can be obtained for strong Kerr nonlinearities at zero frequency. In the frequency and Kerr nonlinearity regime investigated here, nonlinear transfer functions and, thus, non-Gaussian Wigner functions would also occur, but only for strongly mixed input states with a corresponding quadrature noise many orders of magnitude above shot noise. The result would be a mixed non-Gaussian state with an entirely positive Wigner function. In this paper, however, we apply our model to the regime where the noise amplitude is much smaller than the amplitude of the mean-driving field. For every calculation, we have verified that the transfer functions and, thus, the noise transformation are indeed linear, thus, the approximation of Gaussian Wigner functions is appropriate.

Figures 6 and 7 show the noise transformation of a shotnoise-limited driving field at two different Fourier frequencies $\Omega=0.1 \gamma$ and $\Omega=\gamma$, respectively. For both cases, we considered three values for the escape efficiency $\left(\eta_{\mathrm{esc}}=1\right.$, 0.9 , and 0.75$)$. The left nine tiles of Fig. $6(\Omega=0.1 \gamma)$ and Fig. $7(\Omega=\gamma)$ show the Wigner functions obtained for $\eta_{\text {esc }}=1$. One can see that the squeezed quadrature (e.g., the semiminor axis of the noise ellipse) rotates toward the $X_{1}$ quadrature when approaching the critical point $\mathrm{OP}_{6 \text {, crit }}$. When the intracavity power is further increased $\left(\mathrm{OP}_{7}\right.$ and $\left.\mathrm{OP}_{8}\right)$, it rotates in the opposite direction back toward the $X_{2}$ quadrature. For $\Omega=0.1 \gamma$ (Fig. 6, left) being well inside the bandwidth of the KNLC, the squeezed quadrature matches the $X_{1}$ quadrature 

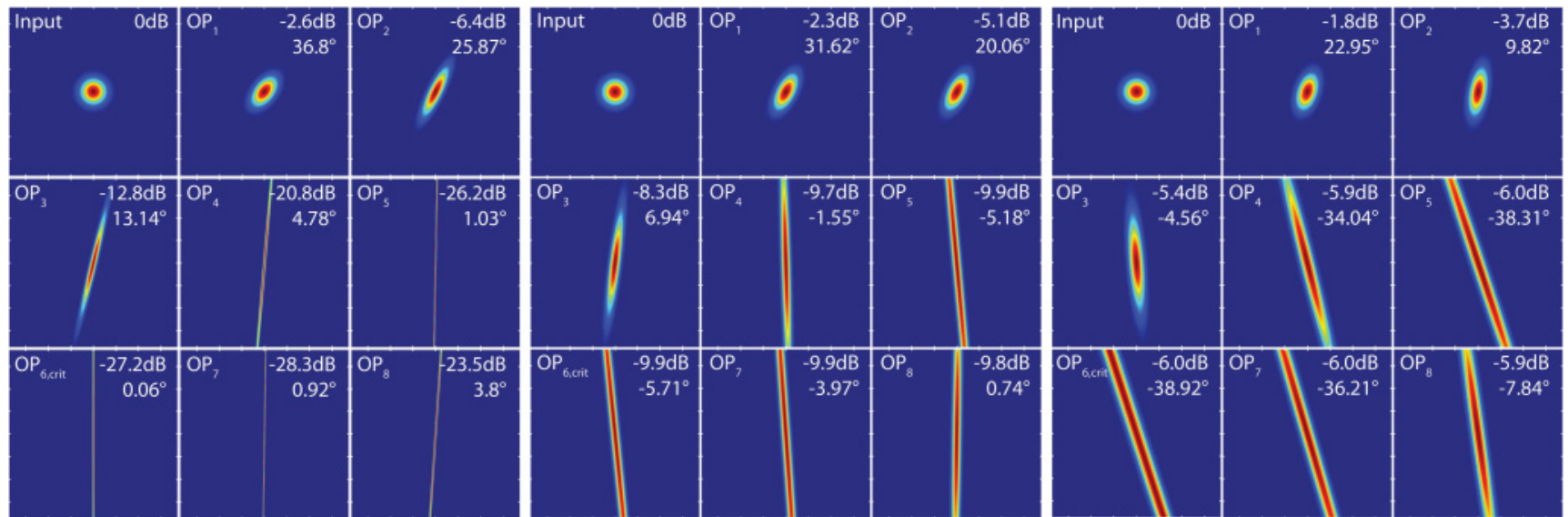

FIG. 6. (Color online) Noise transformations of a (pure) vacuum input state at sideband frequency $\Omega=0.1 \gamma$ for several OPs of the KNLC in terms of Wigner functions. Note that every Wigner function is normalized to its maximum. By that, the size of the colored area increases with the mixedness of the state. For every Wigner function, the angle and the vacuum-normalized variance of the state's lowest noise quadrature is given. (Left) $\eta_{\mathrm{esc}}=1$ (lossless). At $\mathrm{OP}_{6 \text {, crit }}$, the squeezed quadrature matches the amplitude quadrature. (Middle) $\eta_{\mathrm{esc}}=0.9$. The loss changes the phase-space rotation such that the squeezed quadrature matches the $X_{1}$ quadrature at $t w o$ OPs, one close to $\mathrm{OP}_{4}$ and another close to $\mathrm{OP}_{8}$. (Right) $\eta_{\mathrm{esc}}=0.75$. Again, the squeezed quadrature matches the $X_{1}$ quadrature at OPs that depart from the critical point $\mathrm{OP}_{6, \text { crit }}$. The high-optical loss leads to strongly mixed states.

at the critical point $\left(\mathrm{OP}_{6, \text { crit }}\right)$. Considering $\Omega=\gamma$ (Fig 7, left), the squeezed quadrature only approximates the $X_{1}$ quadrature at $\mathrm{OP}_{6, \text { crit }}$. This can be explained by the phase-space rotation caused by a detuned cavity at higher frequencies.

The Wigner functions obtained for $\eta_{\text {esc }}<1$ (middle and right blocks of Figs. 6 and 7) show a qualitatively different behavior of the phase-space rotation. The first essential difference is that, for $\Omega=0.1 \gamma$ in all considered cases, the squeezed quadrature does not match the $X_{1}$ quadrature if the KNLC is operated at its critical point. Again, this fact explains the enhanced noise at low frequencies in the corresponding spectra (refer to Fig. 3). The second difference is that the squeezed quadrature oversteps the $X_{1}$ quadrature at a certain $\mathrm{OP}$ and starts to rotate back again at OPs corresponding to higher intracavity powers. That means the semiminor axis of the noise ellipse coincides with the $X_{1}$ quadrature at two OPs that both depart from the critical point. Both potentially yield a purely optical reduction of laser-power noise or a bright amplitude-squeezed state, respectively. Furthermore, from the comparison of the Wigner functions for $\Omega=0.1 \gamma$ and $\Omega=\gamma$, one can deduce that the squeezing level, or more generally, the noise reduction, can be optimized for a certain frequency by a proper choice of OP. Looking at the left block of Fig. 6, the squeezed quadrature optimally approximates the $X_{1}$ quadrature at a frequency of $\Omega=0.1 \gamma$ if the KNLC is operated at $\mathrm{OP}_{6, \text { crit }}$. In contrast, for $\Omega=\gamma$ (left graph of Fig. 6), the best approach is obviously obtained at an OP being close to $\mathrm{OP}_{5}$.

Figure 8 illustrates the noise transformation of a driving field showing significant classical noise, i.e., being in a highly
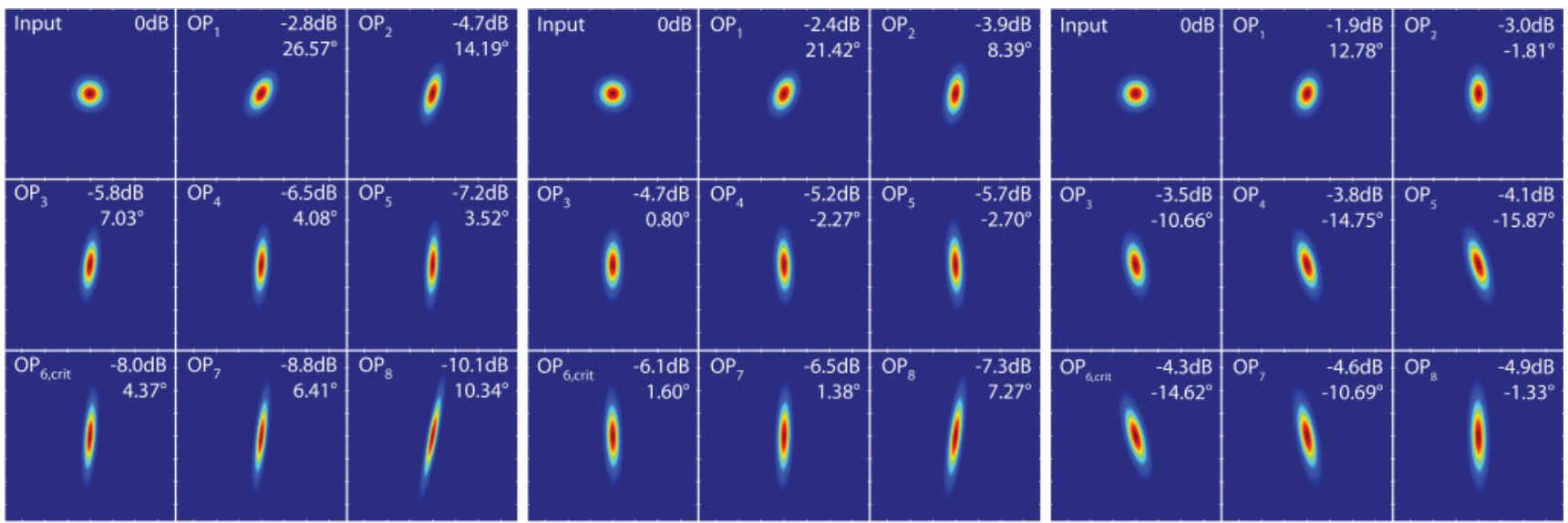

FIG. 7. (Color online) Noise transformations for $\Omega=\gamma$. (Left) $\eta_{\mathrm{esc}}=1$ (lossless). Due to the cavity dispersion at high frequencies, no OP yields amplitude-quadrature squeezing. (Middle) $\eta_{\mathrm{esc}}=0.9$. In contrast to the lossless case, there still exist two OPs at which the squeezed quadrature matches the $X_{1}$ quadrature (one close to $\mathrm{OP}_{3}$ and another close to $\mathrm{OP}_{6, \text { crit }}$ ). (Right) $\eta_{\text {esc }}=0.75$. Due to the high-optical loss, the squeezing is strongly degraded. At $\mathrm{OPs}$ close to $\mathrm{OP}_{2}$ and $\mathrm{OP}_{8}$, the achievable amplitude-quadrature squeezing is about 3 and $4.9 \mathrm{~dB}$, respectively. 


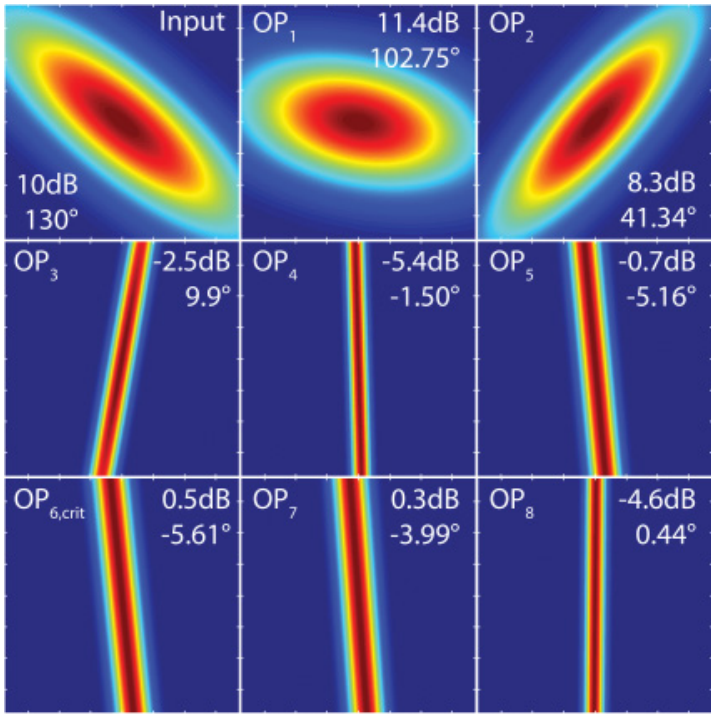

FIG. 8. (Color online) Transformations of a driving field (upper left graph) with an unbalanced noise distribution in phase space. The semimajor axis of the input-noise ellipse corresponds to a noise level of $20 \mathrm{~dB}$ above the shot-noise reference, the semiminor axis to $10 \mathrm{~dB}$ above shot noise. Again, for every Wigner function, the angle and the vacuum-normalized variance of the state's lowest noise quadrature are given. The KNLC has an escape efficiency of $\eta=0.90$. The considered frequency is $\Omega=0.1 \gamma$.

mixed state. This field is constituted just as in the previous section (refer to Fig. 4). The KNLC has an escape efficiency of $\eta_{\text {esc }}=0.9$. The frequency considered is $\Omega=0.1 \gamma$. Again, it can be deduced that there exist two OPs (one close to $\mathrm{OP}_{4}$ and another close to $\mathrm{OP}_{8}$ ) that yield a noise reduction in the $X_{1}$ quadrature of the reflected mean field. Although the driving field exhibits considerable classical noise, a reduction even below shot noise can be achieved. From the comparison with the middle plot of Fig. 6, one can deduce that the orientation of the input field's noise ellipse has a significant influence on the squeezed quadrature of the transformed noise but only at OPs corresponding to intracavity powers smaller than $0.5 P_{\text {res }}$. For higher intracavity powers $\left(\mathrm{OP}_{4}\right.$ to $\left.\mathrm{OP}_{8}\right)$ where the (anti)squeezing levels increase, the squeezed quadrature can be found to be almost the same as in the case of a shot-noise-limited driving field.

\section{OPTIMIZATION IN THE CASE OF LOSS}

From the phase-space representation of the noise transformation (Figs. 6 and 7), we also found that a critical KNLC with internal loss can yield a noise reduction in the amplitude quadrature of the reflected mean field if the OP is chosen properly. By the choice of OP, the noise reduction can be optimized at a certain frequency. Here, we focus on lowsideband frequencies being below the cavity half-linewidth $\gamma$. This regime is of great importance since passive filtering with standard optical cavities can provide a noise reduction only at frequencies above the cavity half-linewidth. Remember that there are two potential OPs yielding a noise reduction at low frequencies. We found that the OP that corresponds to higher intracavity powers yields slightly better noise

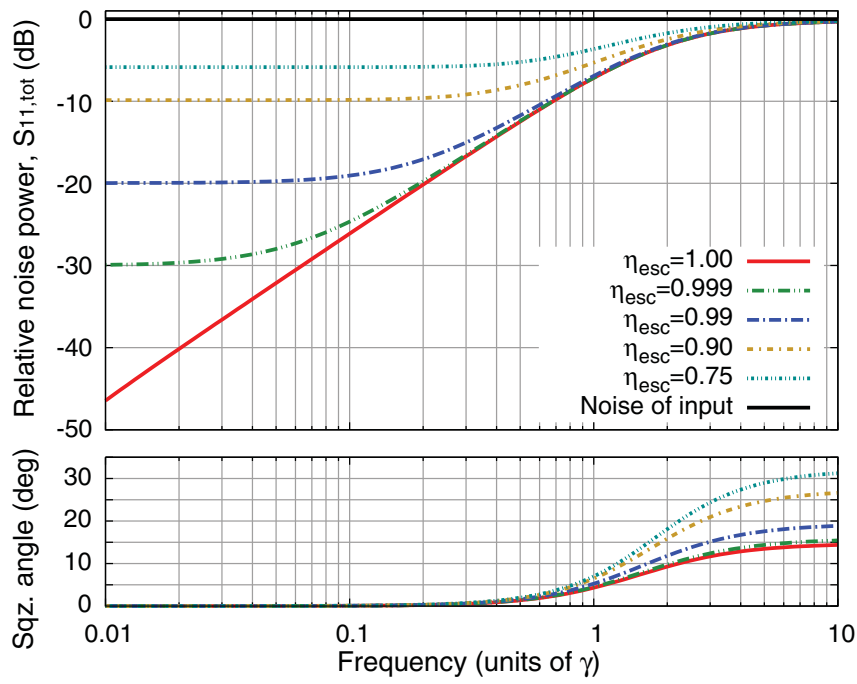

FIG. 9. (Color online) (Top) Amplitude-quadrature noise spectra for a shot-noise-limited input field reflected off the KNLC, which is operated at the optimum operation points, respectively, in order to provide maximum squeezing levels for different intracavity loss values. The squeezing levels approach the value $10 \log _{10}\left(1-\eta_{\text {esc }}\right)$ at low frequencies. (Bottom) Frequency dependence of the squeezing angle (quadrature angle of lowest noise). The OPs are chosen such that this angle is zero at low frequencies in all cases.

reduction. The spectra obtained at the respective optimized OP for a shot-noise-limited driving field are shown in Fig. 9. The lower graph shows the frequency dependence of the quadrature angle yielding the lowest noise. As intended, at low frequencies, it coincides with the amplitude quadrature of the reflected mean field. The achieved squeezing levels (top graph) are solely limited by the intracavity loss and approximate the value $10 \log _{10}\left(1-\eta_{\text {esc }}\right)$ at low frequencies. Although the optimized OPs depart from the critical point, the degradation of the squeezing level is solely caused by the vacuum-noise contribution corresponding to the optical loss.

Similarly, we consider the optimization for the input field showing classical noise as already considered for Figs. 4 and 8. The resulting spectra are shown in Fig. 10. Despite significant driving noise, considerable squeezing levels can still be achieved at low frequencies. In the presence of optical loss, the squeezing level at low frequencies is still limited to a value of $10 \log _{10}\left(1-\eta_{\text {esc }}\right)$, i.e., classical noise of the driving field does not limit the squeezing but its bandwidth. In view of a purely optical passive reduction of laser-power noise in the classical regime, a considerable reduction at low frequencies is always possible, independent of the input field's noise distribution.

\section{COMPARISON WITH EXPERIMENTAL DATA}

Finally, we model the KNLC that was used in Ref. [9] for a passive purely optical reduction of laser-power noise. Its coupling mirror power reflectance was $R_{\mathrm{c}}=0.983$. The intracavity round-trip loss was estimated to $0.5 \%$ resulting in an escape efficiency of $\eta_{\text {esc }} \approx 0.77$. The KNLC's halfbandwidth was about $\gamma \approx 2 \pi \times 4.5 \mathrm{MHz}$. The pump beam was guided through a mode-cleaner ring cavity acting as 

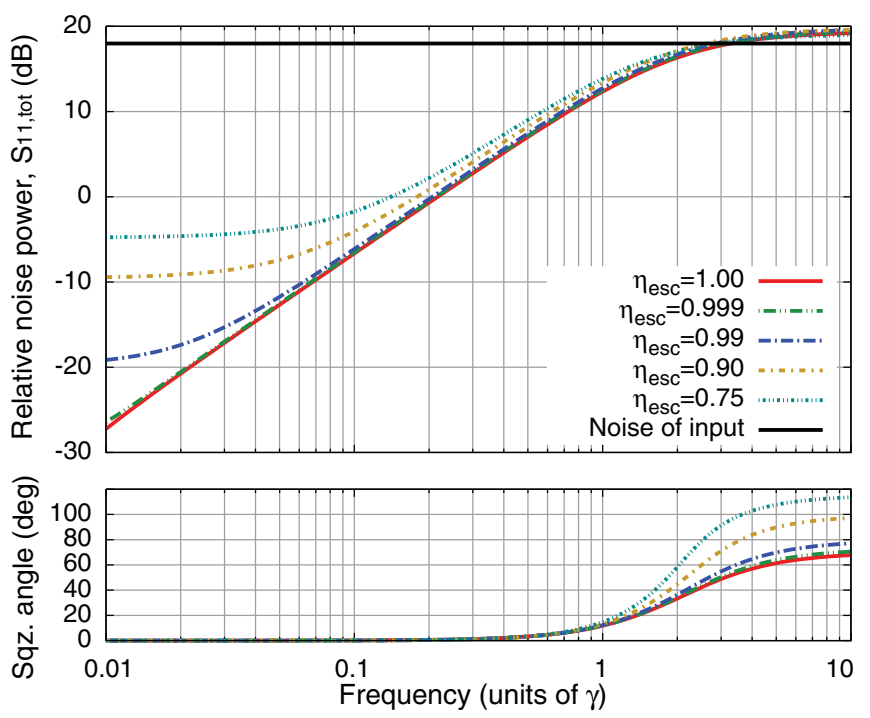

FIG. 10. (Color online) Amplitude-quadrature noise spectra with optimized noise reduction as in Fig. 9 but here, for the input field previously considered in Figs. 4 and 8.

a spatial and low-pass filter (refer to Fig. 2 in Ref. [9]). The filtered beam had a power of about $750 \mathrm{~mW}$ and was coupled to the KNLC. In Ref. [9], it was shown that, for this input power, the KNLC was very close to its critical state. The reflected beam was guided through the mode cleaner a second time and eventually was detected with a photodiode realizing a measurement of the reflected mean field's amplitude-quadrature noise.

In order to model the experimental situation of Ref. [9], first, the characteristics of the input field need to be taken into account. Thus, we have performed a spectral analysis of the power noise of a free-running Nd:YAG laser identical to that used in Ref. [9]. Additionally, we performed a tomographic noise analysis at the frequency of the laser's relaxation oscillation using a balanced homodyne detector. The tomography has revealed that the semimajor axis of the reconstructed noise ellipse deviates by roughly $\vartheta=10^{\circ}$ from the mean field's amplitude quadrature (i.e., $X_{\vartheta-10^{\circ}}=X_{1}$ ). Furthermore, the noise level in the $X_{\vartheta+90^{\circ}}$ quadrature corresponding to the semiminor axis of the noise ellipse has been found to be about $33 \mathrm{~dB}$ below the level in the $X_{\vartheta}$ quadrature. In our noise model, we use the appropriately scaled power-noise measurement as a description of the noise spectrum in the $X_{\vartheta}$ quadrature. Also, in accordance with measurement results, we approximated the noise in the $X_{\vartheta+90^{\circ}}$ quadrature by a cavity pole function having a $1 / f^{2}$ scaling above the laser relaxation oscillation. Furthermore, the orientation of the noise ellipse in phase space is assumed to be $\vartheta=10^{\circ}$ for all frequencies. In order to account for the mode cleaner in the experimental setup, the input-noise data are multiplied by a another pole function describing the mode cleaner's low-pass behavior before the transformation introduced by the KNLC is calculated.

Second, we have verified that the assumption of a linear noise transformation is still appropriate for the power and frequency regimes investigated in Ref. [9]. Although the noise level in the $X_{\vartheta}$ quadrature of the driving field is at the laser relaxation oscillation frequency several orders
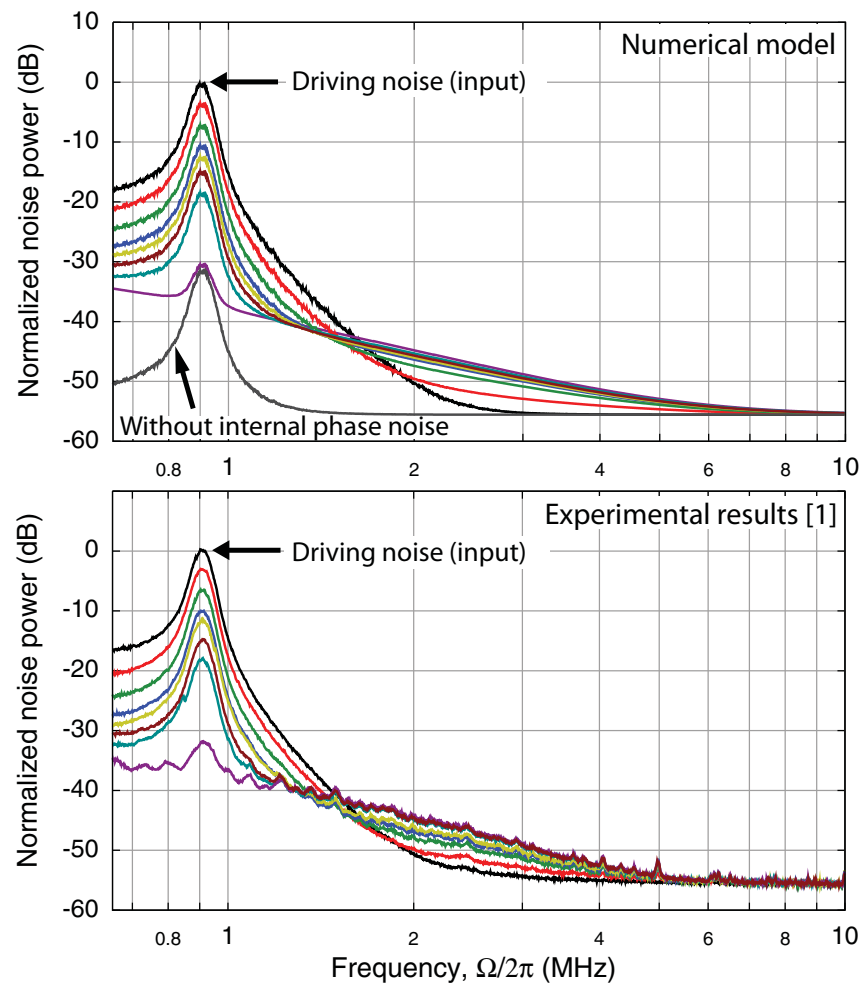

FIG. 11. (Color online) Comparison of our modeled (top) with the measured noise-reduction spectra (bottom) obtained in Ref. [9] for different operation points of the KNLC, normalized to the peak input noise. Our model assumes an additional intracavity $1 / f$ phase noise in order to achieve the qualitative agreement shown. The gray curve in the top graph is obtained instead of the magenta curve if no additional phase noise is considered.

above shot noise, our time-domain simulation does not show any harmonic frequencies. Instead, we find that, for the modeled critical KNLC $\left(P_{\text {in }}=750 \mathrm{~mW}, \eta_{\text {esc }}=0.77, \gamma=\right.$ $2 \pi \times 4.5 \mathrm{MHz}$ ), the nonlinear property of the transfer function in the frequency regime above $\Omega=0.1 \gamma$ only becomes significant for input-noise levels 5 orders of magnitude above the experimentally found peak value. Finally, the field reflected off the critical KNLC is multiplied by the pole function and is attenuated to about $150 \mathrm{~mW}$ in order to model the mode-cleaner low-pass filtering and the photoelectric detection scheme, respectively, used in Ref. [9].

Figure 11 compares the modeled $X_{1}$-noise-reduction spectra (top) with the experimental results (bottom). As in Ref. [9], seven exemplary OPs are considered. They are chosen with respect to the noise reduction at the laser's relaxation oscillation frequency. As already stated in Ref. [9], the experimental noise-reduction spectra show additional noise in the midfrequency range. We have verified that this noise is not due to the Kerr effect since it also appears when the Kerr effect is switched off by rotating the light's polarization by $90^{\circ}$. Presumably, this excess noise is thermally driven (e.g., by thermorefractive noise [23]) as the intracavity powers reached values on the order of $100 \mathrm{~W}$ at a waist size of about $30 \mu \mathrm{m}$. In our model, we assume an intracavity $1 / f$ phase noise that scales with the square of the intracavity power. The strength of this phase noise is fitted such that the magenta curve is 
obtained from the spectrum yielding the best noise reduction (gray curve in Fig. 11). This phase-noise model is used for all other curves. Doing so, it is possible to model noise-reduction spectra being in excellent agreement with the experimental results.

\section{SUMMARY}

We have theoretically investigated the noise transformation of a critical KNLC based on a rigorous treatment in a time-domain simulation. Eventually, we have aimed at the modeling of the passive laser noise reduction with such a cavity, as observed in Ref. [9]. The comparison of our modeled laser-power noise-reduction spectra showed excellent agreement with the experimental results of the work mentioned when adding a $1 / f$ excess phase noise. Our model revealed that the knowledge of both, the optical loss inside the KNLC as well as the phase-space distribution of the input laser noise are crucial for a correct description. In our time-domain simulation of the experimental data of Ref. [9], we have not observed the transformation of input noise to other frequencies, i.e., linearized equations would have been equally valid. We, therefore, restricted our entire theoretical analysis on the influence of optical loss and input-noise phase-space distribution presented here to the regime of linear transfer functions. Our analysis has shown that a KNLC is generally able to provide a noise reduction beyond shot noise, even in the presence of optical intracavity loss and classical driving noise. We have found that the noise reduction in the amplitude quadrature of the reflected mean field can be optimized by the choice of the KNLC operating point. The noise-reduction level at frequencies much smaller than the KNLC's half-bandwidth $\gamma$ has not been found to be limited by the noise of the driving field but solely by the intracavity loss. The bandwidth in which significant squeezing levels can be achieved, however, has turned out to be limited by the amount of classical noise carried by the driving field. We believe that the presented investigations and our numerical model have a high potential to estimate parameters required in existing and future experiments aiming at a purely optical reduction of laser noise or at the squeezing of quantum noise based on a KNLC. A future experiment with considerably stronger driving noise than present in Ref. [9] may show whether our approach also is able to correctly model nonlinear transfer functions and the generation of non-Gaussian noise through a critical KNLC.

\section{ACKNOWLEDGMENTS}

This work was supported by QUEST, the center of excellence of the Leibniz Universität Hannover, and the European Commission's Seventh Framework Programme (FP7/20072013) under Grant No. 211743 (Einstein Telescope Design Study). Henning Rehbein, Nico Lastzka, and Jan Harms are acknowledged for fruitful discussions.
[1] M. Kitagawa and Y. Yamamoto, Phys. Rev. A 34, 3974 (1986).

[2] P. D. Drummond and D. F. Walls, J. Phys. A 13, 725 (1980).

[3] M. J. Collett and D. F. Walls, Phys. Rev. A 32, 2887 (1985).

[4] A. F. Pace, M. J. Collett, and D. F. Walls, Phys. Rev. A 47, 3173 (1993).

[5] H. Rehbein, J. Harms, R. Schnabel, and K. Danzmann, Phys. Rev. Lett. 95, 193001 (2005).

[6] R. Schnabel, N. Mavalvala, D. E. McClelland, and P. K. Lam, Nat. Commun. 1, 121 (2010).

[7] F. Seifert, P. Kwee, M. Heurs, B. Willke, and K. Danzmann, Opt. Lett. 31, 2000 (2006).

[8] P. Kwee, B. Willke, and K. Danzmann, Opt. Lett. 33, 1509 (2008).

[9] A. Khalaidovski, A. Thüring, H. Rehbein, N. Lastzka, B. Willke, K. Danzmann, and R. Schnabel, Phys. Rev. A 80, 053801 (2009).

[10] B. Willke, Laser Photonics Rev. 4, 780 (2010).

[11] G. M. Harry (LIGO Scientific Collaboration), Classical Quantum Gravity 27, 084006 (2010).

[12] M. Punturo et al., Classical and Quantum Gravity 27, 194002 (2010).
[13] S. Reynaud, C. Fabre, E. Giacobino, and A. Heidmann, Phys. Rev. A 40, 1440 (1989).

[14] K. Bergman and H. A. Haus, Opt. Lett. 16, 663 (1991).

[15] R. Dong, J. Heersink, J. F. Corney, P. D. Drummond, U. L. Andersen, and G. Leuchs, Opt. Lett. 33, 116 (2008).

[16] K. S. Zhang, T. Coudreau, M. Martinelli, A. Maître, and C. Fabre, Phys. Rev. A 64, 033815 (2001).

[17] A. G. White, P. K. Lam, D. E. McClelland, H.-A. Bachor, and W. J. Munro, J. Opt. B: Quantum Semiclassical Opt. 2, 553 (2000).

[18] A. Thüring, Ph.D. thesis, Leibniz Universtität Hannover, 2009.

[19] E. Wigner, Phys. Rev. 40, 749 (1932).

[20] M. Frigo and S. G. Johnson, Proc. IEEE 93, 216 (2005), special issue on program Ggneration, optimization, and platform adaptation.

[21] J. Harms, Ph.D. thesis, Leibniz Universtität Hannover, 2006.

[22] N. Lastzka, Ph.D. thesis, Leibniz Universtität Hannover, 2010, Appendix B

[23] V. B. Braginsky, M. L. Gorodetsky, and S. P. Vyatchanin, Phys. Lett. A 271, 303 (2000). 